\title{
Non-surgical periodontal treatment for drug-influenced gingival enlargement: a four-year follow-up case report
}

\author{
Fernanda Srynczyk-da Silva ${ }^{1}$, Giovane Hisse-Gomes², Luis Eduardo Rilling-Nova Cruz ${ }^{1}$, \\ Juan Pablo Aitken-Saavedra ${ }^{3}$, Josué Martos ${ }^{1 *}$
}

\begin{abstract}
1. Department of Semiology and Clinics, School of Dentistry. Federal University of Pelotas, Pelotas, Brazil.

2. Department of Odontology, School of Dentistry. Catholic University of Pelotas, Pelotas, Brazil. 3. Department of Pathology and Oral Medicine. University of Chile, School of Dentistry, Chile.
\end{abstract}

${ }^{*}$ Corresponding Author: Josué Martos, Department of Semiology and Clinics, School of Dentistry | Address: Gonçalves Chaves street. 457, Pelotas, RS 96015-560, Brazil. | Telefax: +55 5332214162. | E-mail: josue.sul@terra.com.br

Work received on 25/04/2020.

Revised work 30/06/2020

Approved for publication on 18/07/2020

\begin{abstract}
Drug-influenced gingival enlargement is considered to be an important side effect related to the use of some medications and often produces important esthetic changes for patients, as well as clinical symptoms such as pain, bleeding, abnormal tooth movement and occlusion problems. Anticonvulsants, immunosuppressants and antihypertensive drugs have been reported as the main inducers of these periodontal disorders. This case report describes the 4-year clinical follow-up of a young patient with a history of excessive gingival enlargement as a consequence of drug therapy for epilepsy (phenytoin and phenobarbital); the nonsurgical periodontal treatment of the excessive gingival enlargement was associated with the replacement of the patient's epilepsy medications with valproic acid. We conclude that intensive mechanical control of bacterial biofilms, instruction on oral hygiene guidelines and behaviors and the substitution of alternative anticonvulsant medications are the best way to control the drug-influenced gingival enlargement.
\end{abstract}

KEY WORDS:

Drug-influenced gingival enlargement; Anticonvulsant; Periodontal treatment.

Int. J. Inter. Dent Vol. 14(2); 184-186, 2021.

\section{INTRODUCTION}

Drug-influenced gingival enlargement is the term currently used to describe the gingival tissue enlargement associated with drugs $^{(1)}$. We have observed an increasing number of drugs associated with this periodontal condition, and these current terms do not accurately reflect the histological composition of the pharmacologically modified gingiva(2).

The new classification of periodontal diseases highlights the importance of bacterial biofilm as a co-factor in the etiology of gingival enlargement associated with drug use. In this new classification, the term "drug-influenced gingival enlargement" is categorized within the group of gingivitis induced by dental plaque ${ }^{(1,3)}$. Despite this, some studies have found no relationship between the accumulation of plaque and excessive gingival enlargement ${ }^{(4,5)}$.

Medications associated with gingival enlargement can be divided into three categories: anticonvulsants, calcium channel blockers and immunosuppressants ${ }^{(1,2)}$. The development of a considerable gingival enlargement was verified in a patient with epilepsy who was prescribed a phenytoin regimen. However, in association with a clinical/surgical treatment the neurologist verified the absence of inflammation and a marked improvement in the patient's gingival architecture after stopping treatment with phenytoin and replacing it with valproic acid(6). In a very similar way a complete regression of generalized gingival enlargement due to the concomitant use of phenytoin and phenobarbital was observed in a young patient after replacement of these drugs with valproic acid and the execution of periodontal treatment ${ }^{(7)}$.

This study reported the 4-year follow-up of a patient with severe gingival enlargement in consequence of drug therapy for epilepsy (phenytoin and phenobarbital) and her nonsurgical periodontal treatment associated with the substitution of medication with valproic acid.

\section{CASE REPORT}

This study was reported in compliance with CARE - Case Report Guidelines ${ }^{(8)}$. A 24-year-old female patient was referred to the Faculty of Dentistry department at our institution for consultation after manifesting intense oral bleeding and difficulty feeding. In anamnesis, it was reported that the patient had a convulsive condition previously diagnosed by a neurologist, and as a consequence, $100 \mathrm{mg}$ phenytoin 3 times a day and $100 \mathrm{mg}$ phenobarbital every 12 hours were used as anticonvulsant drugs. The patient reported that there was no family history of this same clinical

\section{condition.}

Periodontal condition was evaluated during the initial examination and included presence of plaque percentage of affected sites (plaque index $\mathrm{PI})^{(9)}$, percentage of gingival bleeding on probing sites (BOP) periodontal probing depth (PPD) and clinical attachment level (CAL).

Clinically, the patient presented with generalized gingival enlargement with a lobulated aspect and with clinical crowns covered in a large extent, presenting with a buccolingual dimension greater than $3 \mathrm{~mm}$ and a probe depth of $\geq 6 \mathrm{~mm}$ (Figure 1a). The presence of visible plaque and gingival bleeding on probing was observed in all sites where gingival enlargement was observed. The patient used a toothbrush and toothpaste for daily oral hygiene, although in a precarious and difficult way.

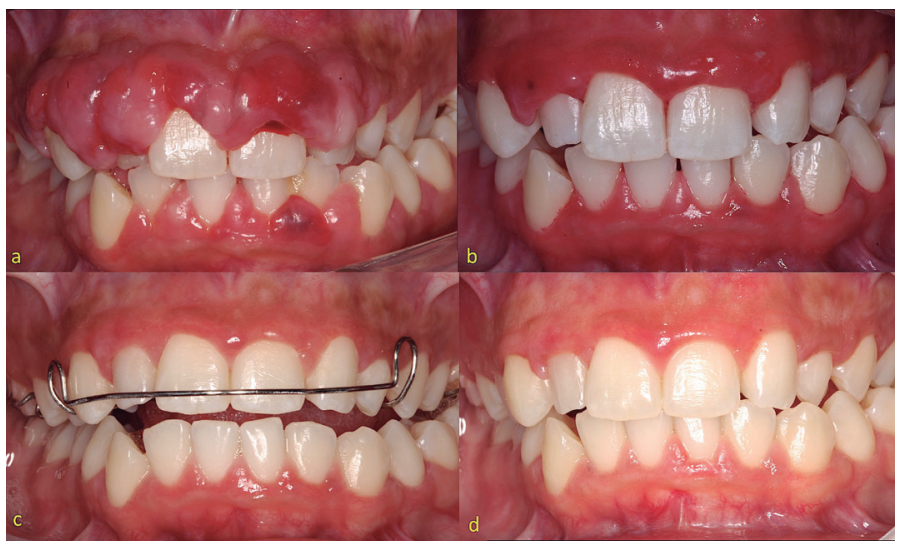

Figure 1: Clinical aspect of the case: (a) before treatment, (b) 12 months, (c) 26 months and (d) 48 months after treatment.

Radiographically there was no evidence of alveolar bone loss, either localized or generalized and periodontal evaluation showed the global visualization of gingival enlargement with some parameters of periodontal insertion (Table 1). The periodontal diagnosis was drug-influenced gingival enlargement, presenting a generalized inflammatory condition, because almost all teeth were affected by gingival inflammation ${ }^{(1)}$. 
Tabla 1: Clinical periodontal parameters at baseline, 12, 26 and 48-months of follow-up.

\begin{tabular}{|l|r|r|r|r|}
\hline & Baseline & 12-months & 26-months & 48-months \\
\hline IP (\%) & 32,03 & 14,06 & 8,59 & $4,68 \%$ \\
BOP (\%) & 32,14 & 19,64 & 13,39 & $4,46 \%$ \\
PPD $\geq 4 m m$ sites & 4,62 & 4,2 & 0 & 0 \\
Mean PPD & $27(24,10 \%)$ & $5(4,46 \%)$ & 0 & 0 \\
number of sites & 1,2 & 1,02 & 1,0 & 0 \\
\hline $\begin{array}{l}\text { CAL } \geq 1 \text { mm sites } \\
\text { Mean CAL }\end{array}$ & $15(13,39 \%)$ & 0 \\
number of sites & $44(39,28 \%)$ & $35(31,25 \%)$ & $15 \%$ & \\
\hline
\end{tabular}

The clinical picture suggested gingival enlargement by the use of anticonvulsant medication and the neurologist was promptly consulted to detail the case and the patient's systemic conditions. Clinical laboratory tests showed normal parameters in the erythrogram present, platelet count $(170.00 / \mathrm{mcl})$, leukogram $(3.800 / \mathrm{mcl})$, blood glucose $(79 \mathrm{mg} / \mathrm{dl})$, as well as for the activated partial thromboplastin time (34s).

The treatment plan was divided into stages; the first one was associated with the systemic control of the convulsive condition with the substitution of phenytoin and phenobarbital previously reported with $125 \mathrm{mg}$ valproic acid three times a day (Depakene, Abbott Laboratories) by her neurologist. The second stage of treatment focused on family orientation and robust supragingival biofilm removal using Gracey curettes, bicarbonate spray and dental floss. In addition to the soft brush, the concomitant use of $0.12 \%$ chlorhexidine digluconate solution twice a day was recommended as an aid in mechanical control during the initial treatment phase.

From the initial period of the therapeutic approach with supragingival biofilm removal followed by subgingival instrumentation associated with switching medication, a positive evolution of the clinical situation could already be seen, reflected in the regression of the gingival enlargement. After reaching a bleeding percentual below $20 \%$, subgingival scaling was performed in weekly sessions for two months (Figure $1 \mathrm{~b}$ ). In this twelvemonth period, the patient had good control of her convulsive condition due to the continuous therapeutic use of valproic acid. Associated with these clinical procedures, some biofilm disclosure sessions were carried out with the use of an evidence-revealing agent for instruction and didactic guidance of the patient.

At the end of twenty-six months of clinical follow-up, the patient performed good mechanical biofilm control as observed by the plaque and gingival bleeding on probing. This condition allowed the initiation of a new treatment phase based on the correction of the palatoversion of the right upper lateral incisor through fixed orthodontic movement followed by installation of a removable containment device (Figure 1c).

Briefly, the periodontal clinical control was achieved with the patient's adherence to the recall system, with weekly, biweekly and monthly followups throughout the treatment, and with evaluating at each consultation the need frequency of upcoming visits in the first 26 months of treatment. After this period, the patient already showed good care for her hygiene and good control in the accumulation of plaque; therefore, the patient required maintenance every three months.

After 48 months of clinical follow-up, although the patient had mild gingival enlargement in the upper lateral incisor, because of a slightly bad dental position, she presented with adequate oral hygiene (Figure 1d). There was no recurrence of gingival enlargement or presence of regions with gingival bleeding after periodontal probing (Figures 2, 3) (Table 1). Oral hygiene maintenance had greatly improved, which resulted in the absence of malodor, thereby significantly increasing the patient's quality of life. The patient has been undergoing intensive medical treatment, with no systemic changes resulting from the change in anticonvulsant drug therapy, and a quarterly maintenance plan, with repeated motivation and information in all clinical sessions, always using positive reinforcement.

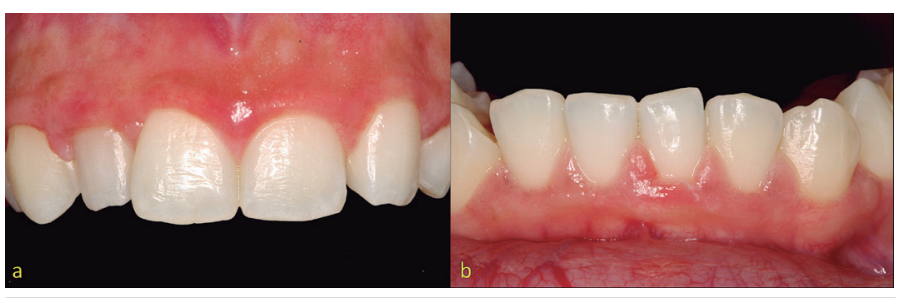

Figure 2. A 48-months periodontal follow-up (a) Maxillary view. (b) Approximate mandibular aspect.



Figure 3. Periodontal probing at 48-month follow-up.

\section{DISCUSSION}

The understanding of epilepsy, its oral manifestations resulting from the medications involved and the periodontal treatment of patients with this clinical condition must be performed by dentists with knowledge about such disorders.

Sodium valproate is a broad-spectrum antiepileptic medication that, according to some authors, is a preferable drug for patients with epilepsy(10). Some antiepileptic drugs (phenytoin, carbamazepine and sodium valproate) have shown a more evident effect on gingival enlargement after its administration; for example, phenytoin manifests more significant gingival enlargement than valproate, which has a low potential|(5,11,12). These findings lead to the conclusion that sodium valproate has a relatively low risk for the development of gingival enlargement and may be an alternative treatment to replace phenytoin ${ }^{(11)}$. These findings are in line with what was observed in this clinical case report, where the substitution between these drugs seems to have been pivotal in the resolution of the patient's gingival enlargement.

In a general way the pathogenesis of drug-influenced gingival enlargement can be considered in a multifactorial way involving an interaction of several factors such as drug metabolism with gingival fibroblasts, age, genetic predisposition and even inflammatory changes ${ }^{(13)}$. In this respect, the new classification of periodontal diseases exemplifies the importance of bacterial biofilm as one of the essential factors for drug-influenced gingival enlargement, however without specifying this relationship with valproic acid derivatives ${ }^{(1)}$. In our clinical case, the presence of dental biofilm was an evident observation in the initial consultations, as verified by the visible plaque index and certainly was a determinant for the excessive gingival enlargement observed. For this reason, an intense regime of mechanical control of bacterial plaque, both professional, through consultations of supragingival scraping and frequent hygiene guidance, as well as by the patient, following these guidelines, were fundamental for the resolution of the inflammatory process and consequent reduction of gingival enlargement.

Multidisciplinary intervention and early management of drug-influenced gingival enlargement offer the best opportunity for positive results ${ }^{(7,13,14)}$. It is important to highlight the relevance of periodontal maintenance therapy, and the need for periodic and permanent monitoring of this type of patient, in preserving periodontal health in the long term. This observation justifies our conduct related to the high frequency of referral consultations for our patient.

According to the literature, the intervals for repeat consultations or calls for support treatment vary greatly according to each author and their research. This variation can range from 2 weeks to 18 months, but for individuals with a history of periodontitis, it is suggested that these consultations be performed at intervals of less than 6 months ${ }^{(15)}$. Despite this, this periodicity must be individualized, meeting the needs of each patient ${ }^{(15)}$. In our clinical case, due to the patient's susceptibility to gingival enlargement and the consequent greater difficulty in performing oral hygiene behaviors, we perform maintenance sessions every 30 days in the first year and every 3 months after that period. Our procedures included behavioral change instruction, such as oral hygiene guidance, supragingival biofilm removal and dental prophylaxis, as recommended by the American Academy of Periodontology ${ }^{(15)}$.

It is important to point out that non-surgical periodontal treatment presented should have been reviewed in the distal area of papilla of the right maxillary lateral incisor after orthodontic treatment. Even with orthodontic stabilization, a small excess of gingival tissue in the papillary 
area remained remnant, which can constitute an area with difficulty in routine dental cleaning by the patient. The present limitation can also be duly corrected with a periodontal surgical intervention.

We emphasize the importance that neurologists perceive and detect the potential effects of gingival enlargement induced by antiepileptic drugs and their characteristics to prevent, diagnose and successfully administer treatment alternatives ${ }^{(16)}$. This knowledge will also allow the patient to be referred to the dental surgeon who will be able to carry out preventive measures through the removal of the supra- and subgingival biofilm and by offering oral hygiene guidance. This multiprofessional approach will enable a more adequate resolution of the consequences of the disease and its treatment, as can be well observed by the clinical results obtained in the clinical case presented.

\section{CONCLUSION}

The intensive mechanical control of the bacterial biofilm, the oral hygiene guidelines and the substitution of medications with other alternatives have been shown to be effective in controlling the druginfluenced gingival enlargement. The periodontal maintenance therapy implemented was demonstrated to be adequate for maintaining periodontal health after 4 years of follow-up.

\section{DECLARATION OF CONFLICT OF INTEREST AND FUNDING} SOURCE

\section{Ethical disclosures}

Protection of human and animal subjects.

The authors declare that no experiments were performed on humans or animals for this investigation.

\section{Confidentiality of data}

The authors declare that they have followed the protocols of their work center on the publication of patient data.

\section{Right to privacy and informed consent}

The authors must have obtained the informed consent of the patients and/or subjects mentioned in the article. The author for correspondence must be in possession of this document.

\section{CLINICAL RELEVANCE}

The scientific justification for this report was to demonstrate that the intensive mechanical control of the bacterial biofilm, the oral hygiene guidelines and the substitution of medication have been shown to be effective in controlling the drug-influenced gingival enlargement after 4-years follow-up. Clinical recommendations to be adopted would be interdisciplinary action and the need for periodontal maintenance to perpetuate the health acquired after the active phase of periodontal treatment. Reinforcing that maintenance is an extension of periodontal treatment, to allow patients previously treated for periodontal disease to be included in this phase in the long term, preventing disease recurrence and progression.

\section{CONFLICT OF INTEREST STATEMENT}

None declared.

\section{Reference}

1. Murakami S, Mealey BL, Mariotti A, Chapple ILC. Dental plaque-induced gingival conditions. J Periodontol. 2018;89(Suppl 1):S17-S27.

2. Dongari-Bagtzoglou A. Drug-associated gingival enlargement. J Periodontol. 2004;75:1424-31.

3. Chapple ILC, Mealey BL, Van Dyke TE, Bartold PM, Dommisch H, Eickholz P, et al. Periodontal health and gingival diseases and conditions on an intact and a reduced periodontium. J Clin Periodontol. 2018;45:S68-S77.

4. Nishikawa S, Nagata T, Morisaki I, Oka T, Ishida H. Pathogenesis of druginduced gingival overgrowth. A review of studies in the rat model. J Periodontol. 1996;67:463-71.

5. Suneja B, Chopra S, Thomas AM. A clinical evaluation of gingival overgrowth in children on antiepileptic drug therapy. J Clin Diagn Res. 2016;10:ZC32-6.

6. Pette GA, Siegel MA, Parker WB. Gingival enlargement. J Am Dent Assoc. 2014:142(11):1265-68.

7. Miguelis TMF, Pinto KVA, Nova Cruz LER, Saavedra JP, Martos J. Systemic and clinical treatment of gingival hyperplasia associated with use of anticonvulsant. Rev Clin Periodoncia Implantol Rehabil Oral. 2019;12(1);50-2.

8. Gagnier JJ, Kienle G, Altman DG, et al. The CARE guidelines: consensus-based clinical case report guideline development. J Diet Suppl. 2013;10(4):381-90.
9. Ainamo J, Bay I. Problems and proposals for recording gingivitis and plaque. Int Dent J. 1975;25(4):229-35.

10. Brodie MJ, Dichter MA. Antiepileptic drugs. N Engl J Med. 1996;334:168-75 11. Seymour RA, Smith DG, Turnbull DN. The effects of phenytoin and sodium valproate on the periodontal health of adult epileptic patients. J Clin Periodontol. 1985;12:413-9.

12. Nevitt SJ, Marson AG, Weston J, Tudur Smith C. Sodium valproate versus phenytoin monotherapy for epilepsy: an individual participant data review. Cochrane Database Syst Rev. 2018;9:CD001769.

13. Patil RB, Urs P, Kiran S, Bargale SD. Global developmental delay with sodium valproate-induced gingival hyperplasia. BMJ Case Rep. 2014;1-4.

14. González AC, Castañeda LN, Romano PR, Schneider AR, Toro MAF, Hofer FD. Agrandamiento gingival por ciclosporina: reporte de un caso. Rev Clin Periodoncia Implantol Rehabil Oral. 2016;9:226-30.

15. Cohen RE. Position paper: Periodontal maintenance. J Periodontol. 2003;74:1395-401.

16. Lin k, Guilhoto LMFF, Yacubian EMT. Drug-induced gingival enlargement - Part II. Antiepileptic drugs: not only phenytoin is involved. J Epilepsy Clin Neurophysiol. 2007;13:83-8 